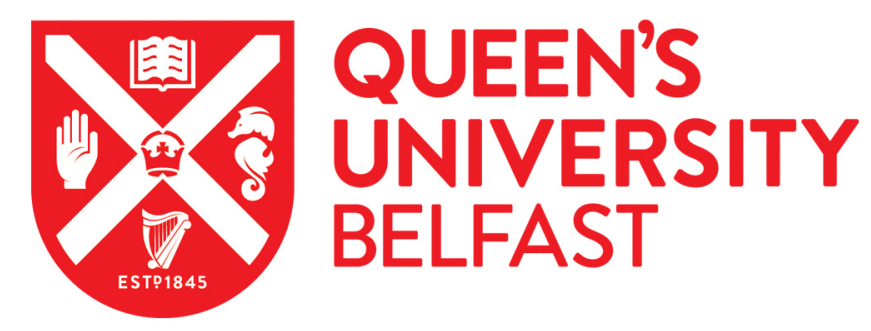

\title{
Towards a Si/GaAs Based Flat-Panel Quasi-Optical Metasurface Antenna with Switchable Beam Characteristics
}

Yurduseven, O., Lee, C., Gonzalez-Ovejero, D., Ettorre, M., Sauleau, R., Fusco, V., Chattopadhyay, G., \& Chahat, N. (2020). Towards a Si/GaAs Based Flat-Panel Quasi-Optical Metasurface Antenna with Switchable Beam Characteristics. In 2020 14th European Conference on Antennas and Propagation (EuCAP) Institute of Electrical and Electronics Engineers Inc.. https://doi.org/10.23919/EuCAP48036.2020.9135231

Published in:

2020 14th European Conference on Antennas and Propagation (EuCAP)

Document Version:

Peer reviewed version

Queen's University Belfast - Research Portal:

Link to publication record in Queen's University Belfast Research Portal

Publisher rights

Copyright 2020 IEEE. This work is made available online in accordance with the publisher's policies. Please refer to any applicable terms of use of the publisher.

\section{General rights}

Copyright for the publications made accessible via the Queen's University Belfast Research Portal is retained by the author(s) and / or other copyright owners and it is a condition of accessing these publications that users recognise and abide by the legal requirements associated with these rights.

Take down policy

The Research Portal is Queen's institutional repository that provides access to Queen's research output. Every effort has been made to ensure that content in the Research Portal does not infringe any person's rights, or applicable UK laws. If you discover content in the Research Portal that you believe breaches copyright or violates any law, please contact openaccess@qub.ac.uk. 


\title{
Towards a Si/GaAs Based Flat-Panel Quasi-Optical Metasurface Antenna with Switchable Beam Characteristics
}

\author{
Okan Yurduseven ${ }^{1}$, Choonsup Lee ${ }^{2}$, David Gonzalez-Ovejero ${ }^{3}$, Mauro Ettorre ${ }^{3}$, Ronan Sauleau ${ }^{3}$,Vincent Fusco ${ }^{1}$, \\ Goutam Chattopadhyay ${ }^{2}$ and Nacer Chahat $^{2}$ \\ ${ }^{1}$ Centre for Wireless Innovation, Institute of Electronics, Communications and Information Technology, School of Electronics, \\ Electrical Engineering and Computer Science, Queen's University Belfast, Belfast BT3 9DT, United Kingdom \\ okan.yurduseven@qub.ac.uk \\ ${ }^{2}$ NASA Jet Propulsion Laboratory, California Institute of Technology, Pasadena, CA 91109, USA \\ ${ }^{3}$ University of Rennes, CNRS, IETR - UMR 6164, F-35000, Rennes, France
}

\begin{abstract}
This paper presents a III/V compound silicon (Si) and gallium arsenide (GaAs) metasurface antenna to achieve beam-forming at $94 \mathrm{GHz}$. The metasurface antenna consists of a flat-panel system architecture and works in a holographic manner. The guided mode launched into the $\mathrm{Si}$ layer is converted to a planar wave-front using a quasi-optical pillbox feeding architecture, significantly simplifying the design process of the metasurface antenna. It is demonstrated that the developed metasurface can switch its radiation pattern in the azimuth plane by selectively activating between multiple input ports while beam forming in the elevation plane can be achieved by actively tuning the metamaterial elements forming the metasurface aperture. The simulated reflection coefficient patterns suggest an impedance matching better than $-15 \mathrm{~dB}$ at all input ports is achieved at $94 \mathrm{GHz}$. The radiation patterns of the antenna clearly demonstrate the $3 D$ beam scanning capability of the presented metasurface antenna $\left(\theta=0^{\circ} / 15^{\circ}\right.$, $\varphi=0^{\circ} / \pm 0^{\circ}$ ) at millimeter-wave frequencies.
\end{abstract}

Index Terms-antennas, metasurface, metamaterial, silicon, gallium arsenide, beam forming, millimeter-wave.

\section{INTRODUCTION}

Antennas with reconfigurable radiation characteristics have been in the forefront of research conducted within the antennas and propagation community for several decades. Synthesizing a desired wave-front on an antenna aperture to produce a radiation pattern of interest has been conventionally realized using the phased-array technique as the beam-shaping mechanism [1, 2]. Typically, a phased array antenna consists of an array of individual radiating elements that sample the aperture at a Nyquist limit. Each individual antenna within the composite phased array aperture requires a phase-shifting circuit, and also a power amplifier to compensate for the insertion losses of the phase shifting circuits. As a result, although beam-shaping capability can be achieved to an excellent fidelity, phased array antennas typically exhibit a complex physical hardware architecture and consume a considerable amount of power. These cost and power constraints have historically limited the use of the phased-array technology mostly to militarybased applications.
Metamaterials are artificial, sub-wavelength structures that can be engineered to achieve electrical properties that are not available in nature, with applications ranging from negative-index media to invisibility cloaks [3-6] to name a few. An array of metamaterial elements (or meta-elements) sampled at a sub-wavelength limit across a planar, twodimensional (2D) surface forms a metasurface aperture [7]. Metasurface antennas can offer the advantage of synthesizing a desired amplitude and phase profile at the antenna aperture without the need for the phase shifting technology. This property enables metasurface antennas to exhibit on-par radiation characteristics with phased arrays but at a fraction of cost and power consumption requirements. Moreover, metasurface antennas can be designed to have a flat-panel system architecture [8-16], and thus, has found numerous applications from radar imaging [17-26] to wireless power transfer [27-29]. In this paper, we present our most recent advancements towards realizing the first III-V silicon ( $\mathrm{Si}$ ) - Gallium Arsenide (GaAs) flat panel reconfigurable metasurface antenna at millimeter-wave frequencies.

\section{METASURFACE ANTENNA}

\section{A. Pillbox Feeding Structure}

The metasurface antenna consists of a dual-layer pillbox feeding structure as depicted in Fig. 1. The pillbox design is a well-known architecture in the literature and enables a planar wavefront to be achieved in a quasi-optical manner [30]. The dielectric material for the bottom layer of the pillbox feed is silicon (Si), $\varepsilon_{r}=11.9, \rho=10 \mathrm{k} \Omega . \mathrm{cm}$ and $350 \mu \mathrm{m}$ thick, while the dielectric layer for the top layer is gallium arsenide (GaAs), $\varepsilon_{r}=12.8$ and $175 \mu \mathrm{m}$ thick. The pillbox has a multi-port feeding architecture, consisting of three coplanar-waveguide (CPW) feeding ports.

The architecture of the feeding ports is depicted in detail in Fig. 2. As shown in Fig. 2, the wave is launched into the Si substrate by the CPW feed ports, which are connected to 1 $\mathrm{mm}$ end launch PCB connectors for the fabricated protype of the antenna. The guided mode launched by the CPW feeding 
ports in the Si layer is radiated by surface-integratedwaveguide (SIW) horn antennas etched into the Si substrate.

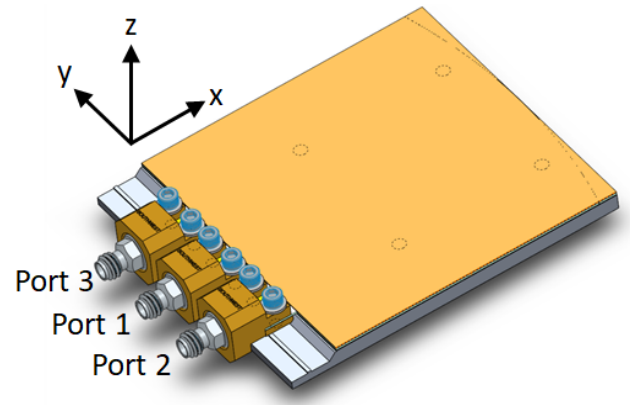

(a)

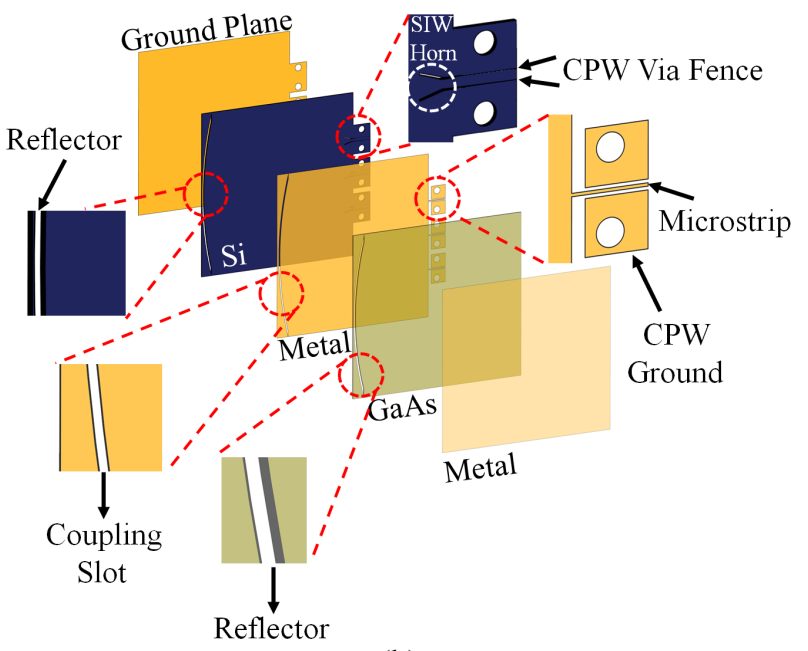

(b)

Fig. 1. Pillbox feeding architecture (a) overall view of the pillbox (b) layer-by-layer depiction of the pillbox (without the metasurface etched onto the top metal layer). Azimuth: $y z$-plane, elevation: $x z$-plane.

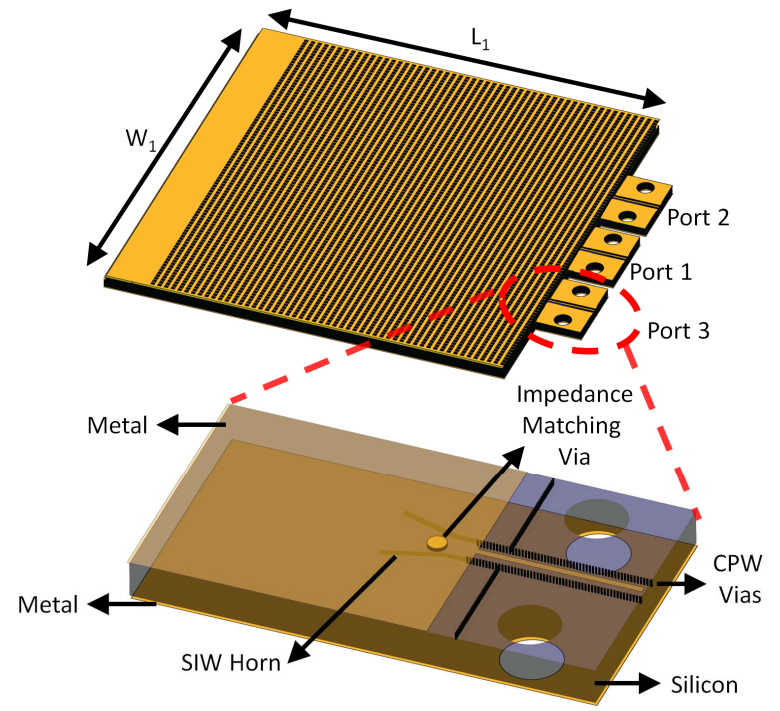

Fig. 2. CPW feeding structure of the metasurface antenna. $W_{1}=50 \mathrm{~mm}, L_{1}=50 \mathrm{~mm}$.

The radiated wavefront by the SIW horn antenna has a cylindrical profile as shown in Fig. 3.

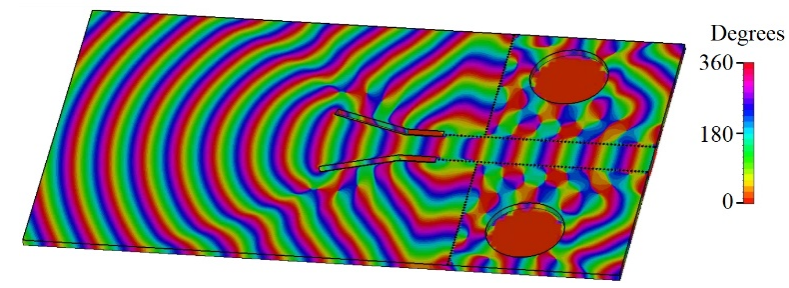

Fig. 3. Phase of the guided mode pattern launched through port 1 (center) into the Si substrate at $94 \mathrm{GHz}$.

The fabricated feeding port structure with the CPW - SIW conversion is shown in Fig. 4. As can be seen in Fig. 4, the SIW horn antenna has a pin (diameter $=50 \mu \mathrm{m}$ and thickness $=85 \mu \mathrm{m}$ ) embedded inside the waveguide, optimizing the impedance match seen at the input of the $\mathrm{CPW}$. The pin is gold coated (coating thickness is $1 \mu \mathrm{m}$ ).

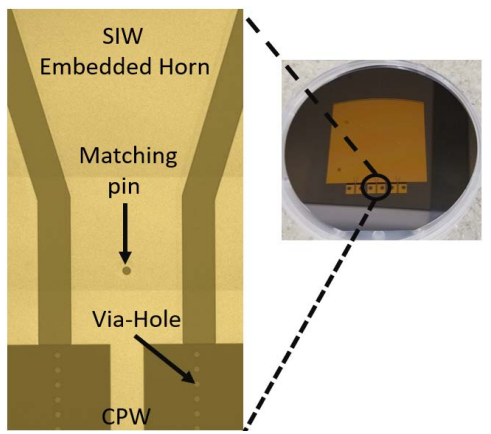

Fig. 4. CPW to SIW transition. The rim of the horn antenna etched within the Si substrate can be seen.

The pillbox feeding structure of the metasurface consists of two layers, Si (bottom) and GaAs (top), with the two layers sharing a reflector rim etched on the opposite side of the feeding ports. As depicted in Fig. 5, the guided mode launched into the $\mathrm{Si}$ layer (with a cylindrical wavefront) propagates towards and reflects off of the reflector rim. The reflected signal couples to the top GaAs layer, which then propagates in the opposite direction. The coupled wave in GaAs layer has a planar wavefront and leaks out through the meta-elements into free-space as it propagates, generating the desired radiation pattern of interest.

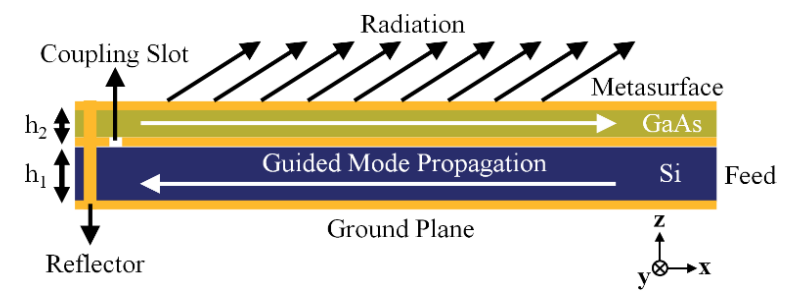

Fig. 5. The coupling of the guided mode launched in Sil substrate (bottom) to the GaAs substrate (top) and its radiation into free-space through the metasurface layer. Dimensions: $h_{1}=350 \mu \mathrm{m}$ and $h_{2}=175 \mu \mathrm{m}$.

The guided mode wavefront inside the $\mathrm{Si}$ and GaAs layers are shown in Fig. 6. A picture of the Si wafer during the fabrication process of the pillbox design is shown in Fig. 7. 


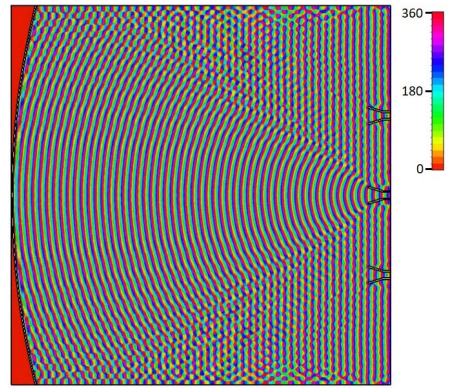

(a)

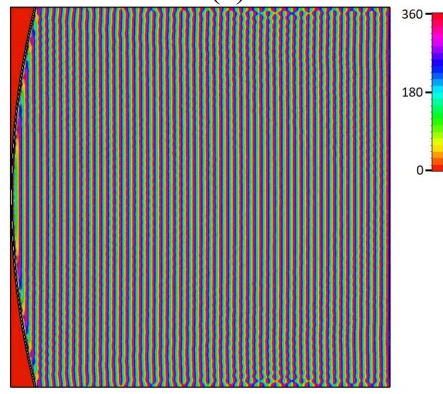

(b)

Fig. 6. Launched E-field guided mode wave patterns at 94 $\mathrm{GHz}$ (phase) inside (a) Si substrate (b) GaAs substrate.

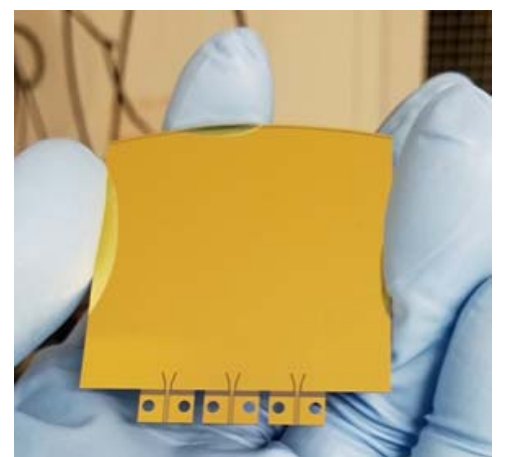

Fig. 7. Picture of the Si wafer in the middle of through etch.

\section{B. Metasurface Design}

The quasi-optical metasurface antenna operates in a holographic manner. Within the holographic framework, the guided mode pattern launched by the feeding port works as a reference-wave. In order to examine the design process, let us consider a one-dimensional (1D) microstrip transmission line loaded with complementary, slot-shaped meta-elements as depicted in Fig. 8. The launched magnetic field (H-field) along this transmission line can be given as follows:

$$
\vec{H}_{r e f}=H_{0} e^{-j \beta x} \hat{y}
$$

Because the 1D metasurface architecture depicted in Fig. 8 consists of complementary slot-shaped meta-elements, we consider the $\mathrm{H}$-field as the guided mode reference exciting the meta-elements. From antenna theory, the array factor (AF) for the presented antenna can be calculated as [31]:

$$
A F(\theta)=\sum_{i=1}^{N} \alpha_{m, i}(w) e^{-j \beta x_{i}} e^{-j k x_{i} \sin \theta \cos \varphi}
$$

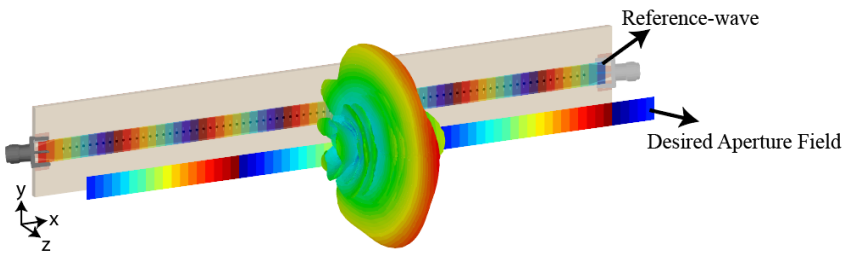

Fig. 8. Depiction of a 1D microstrip transmission line loaded with complementary metamaterial elements.

In (2), $i$ refers to the meta-element number, $w$ is the frequency, $\beta$ is the wavenumber in dielectric and $k$ is the wavenumber in free space. Although sharing a similar AF definition, the presented metasurface concept differs from conventional array antennas in that the aperture is sampled at a subwavelength limit, in comparison to the regular $\lambda_{0} / 2$ limit where $\lambda_{0}$ is the free-space wavelength. This aspect of the presented design enables the guided mode to be modeled as a continuous hologram while ensuring that the antenna architecture does not rely on the phase shifting technology to achieve beam forming.

In (2), $\theta$ and $\varphi$ indicate the beam-forming direction $\left(\varphi=0^{\circ}\right.$ for the 1D example and hence $\cos (\varphi)=1$ can be dropped) while $\alpha$ denotes the polarizability of the meta-elements. The radiation from a sub-wavelength sized complementary metamatetrial element can be modeled as a magnetic dipole with dipole moment of the element can be given as $m=\alpha H$ [31]. Therefore, using the amplitude and phase variation caused by the polarizability distribution of the metaelements, the guided mode (reference-wave) can be modulated into a desired waveform on the metasurface aperture to radiate a radiation pattern of interest in the farfield. This radiation pattern synthesis process does not require any phase-shifting circuit and power amplifiers.

From (2), in order to maximize the array factor in $\theta$, it is evident that the polarizability distribution of the metaelements should be chosen as

$$
\alpha_{m, i}(w)=e^{j \beta x_{i}} e^{j k x_{i} \sin \theta}
$$

Interacting (1) and (2), one can calculate a continuous holographic phase grating with a phase range of $0-2 \pi$. This ideal phase grating for a given $\theta$-direction is retrieved using the metasurface layer with element polarizabilities defined in (3) being applied to the reference-wave in (1). This selection performs surprisingly well despite the assumption that the guided mode holographic reference is not perturbed by the scattering from the meta-elements (weakly-scattered regime) and the Lorentzian limited $0-\pi$ radians achievable phase range of the complementary meta-elements. In this work, we realize the desired aperture wave-front by sampling the antenna aperture at phased-matched points using weakly-scattering, weakly-resonant, slot-shaped metaelements as the radiating metamaterial element type.

As shown earlier in Fig. 5(b), analyzing the phase-front of the coupled wave in the GaAs layer, it is evident that the guided mode inside in GaAs has a planar wavefront. This observation ensures that the metasurface has, ideally, a 
constant phase value for each column, suggesting that we can design a 2D metasurface using the mathematical foundation laid out for the $1 \mathrm{D}$ model. This aspect of the proposed design also brings the advantage that the biasing of the meta-elements across the metasurface can be achieved using a single biasing line per column as opposed to individually biasing the metamaterial elements to achieve dynamic beam forming. This can significantly simplify the biasing circuitry required to tune the meta-elements across the metasurface aperture. The designed metasurface antenna is depicted in Fig. 9 while the fabricated prototype by the NASA's Jet Propulsion Laboratory is shown in Fig. 10.

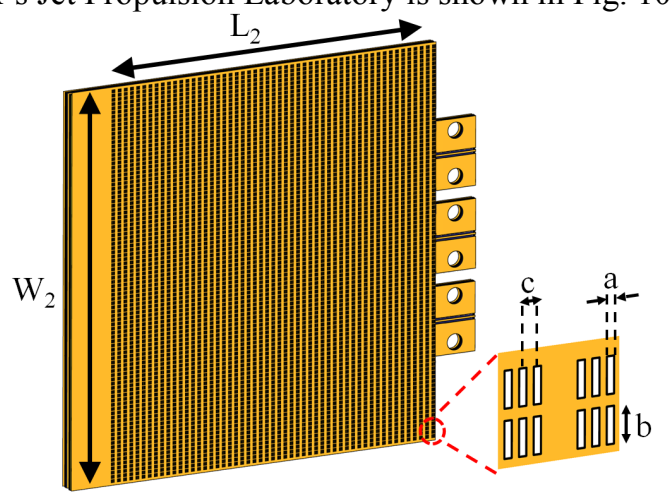

Fig. 9. Metasurface antenna design. $L_{2}=44.5, \quad W_{2}=49$, $a=0.085, b=$ tapered from 0.38 to $0.4, c=0.17$. Units: $\mathrm{mm}$.

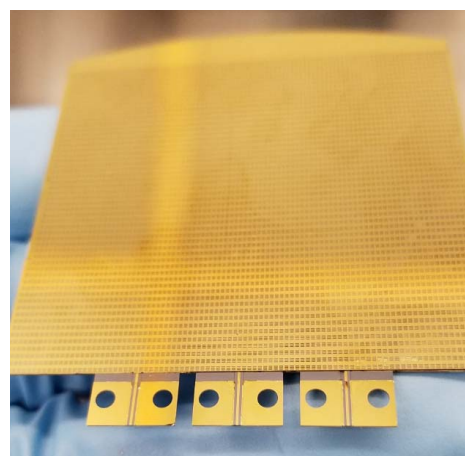

Fig. 10. Fabricated $\mathrm{Si} / \mathrm{GaAs}$ quasi-optical metasurface antenna design with the etched meta-elements.

\section{RESULTS}

The simulated reflection coefficient patterns at the input ports $\left(S_{11}\right.$ and $\left.S_{22}\right)$ are shown in Fig. 11. It should be noted that due to structural symmetry, $S_{33}$ is identical to $S_{22}$ and is not shown in Fig. 11. Fig. 11 also shows the cross-coupling S-parameter results in between multiple ports $\left(\mathrm{S}_{12}\right.$ and $\left.\mathrm{S}_{23}\right)$. Analyzing Fig. 11, it is evident that the metaurface antenna is well matched, with the $S_{11}$ and $S_{22}$ levels remaining below $15 \mathrm{~dB}$ at $94 \mathrm{GHz}$. As shown in Fig. 11, the cross-coupling levels between the input ports are $-28 \mathrm{~dB}$ for $\mathrm{S}_{21}$ (and hence for $\mathrm{S}_{31}$ ) and $-11 \mathrm{~dB}$ for $\mathrm{S}_{23}$ at $94 \mathrm{GHz}$. However, the input ports of the presented quasi-optical metasurface antenna are to be activated not simultaneously but sequentially. Because a simultaneous excitation of the input ports is not required, this coupling does not exhibit a challenge for the presented metasurface antenna architecture.

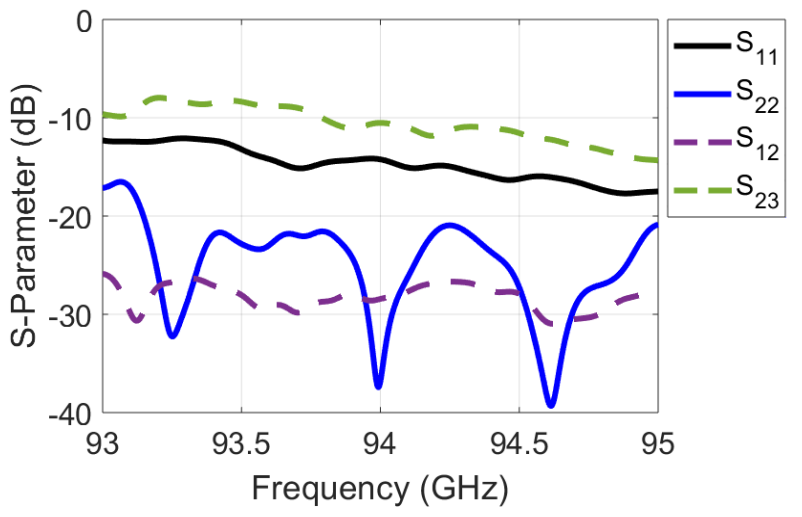

Fig. 11. Simulated S-parameter results for the metasurface antenna for multiple input ports.

The simulated radiation patterns of the metasurface antenna are shown in Fig. 12. When fed through the first port, the metasurface radiates at $\theta=15^{\circ}, \varphi=0^{\circ}$ with a directive gain $31.9 \mathrm{dBi}$, resulting in an aperture efficiency of $59 \%$. This beam control along the elevation plane is governed by (2) and can be dynamically tuned by controlling the coupling responses of the meta-elements using active elements, such as PIN diodes [32, 33]. Switching to port 2 and port 3 enables the metasurface to reconfigure its radiation pattern in the azimuth plane, with the metasurface antenna radiating at $\theta=15^{\circ}, \varphi=90^{\circ}$ when port 2 is activated and at $\theta=15^{\circ}, \varphi=-90^{\circ}$ when port 3 is activated. The half-power-beamwidth (HPBW) values are recorded to be $5.5^{\circ}$ for ports 2 and 3 and $4.4^{\circ}$ for port 1 whereas the directivity difference between these scenarios is reported to be $3.1 \mathrm{~dB}$. It should be noted that due to the frequency-dependence leaky-wave characteristics of the guided-mode, sweeping the operating frequency results in beam-scanning in the elevation direction. This feature can be useful for applications where the aperture wave-front synthesis is required to be achieved in an entirely passive manner. This feature of the antenna will be demonstrated in future work.

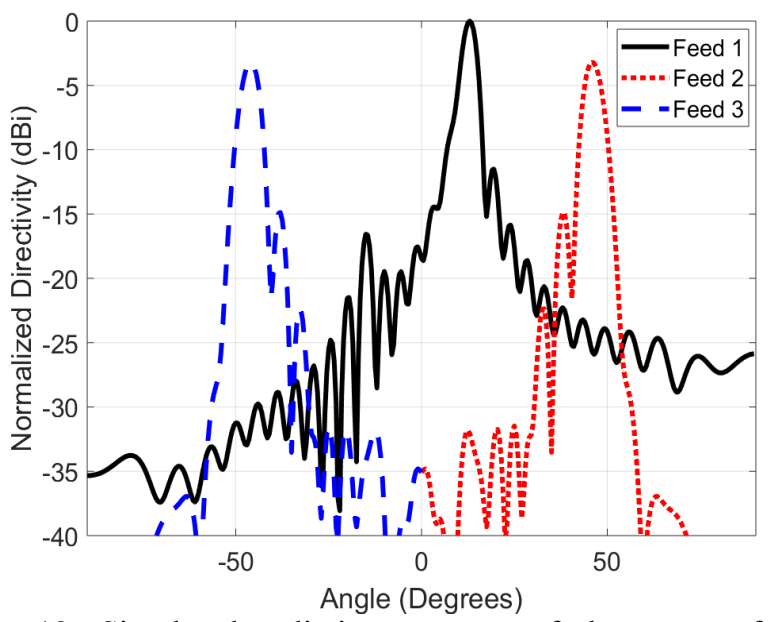

Fig. 12. Simulated radiation patterns of the metasurface antenna at $94 \mathrm{GHz}$ when fed through ports 1-3. Feed 1 (Eplane, $\varphi=0^{\circ}$ ). Feed 2 (H-plane, $\varphi=90^{\circ}$ ) and feed 3 (H-plane, $\varphi=-90^{\circ}$ ). 


\section{CONCLUSION}

We have demonstrated a $\mathrm{Si} / \mathrm{GaAs}$ based metasurface antenna for beam forming at millimeter-wave frequencies. The antenna synthesizes a radiation pattern of interest using the holographic principle by means of modulating a guided mode reference into an aperture wavefront of interest. The developed metasurface can achieve 3D beam steering by appropriately tuning the meta-elements and switching between multiple input ports. Measurements of the fabricated antenna are currently underway at the NASA's Jet Propulsion Laboratory. Future work will look at techniques to develop diodes on $\mathrm{Si} / \mathrm{GaAs}$ substrate to achieve dynamic modulation of the metasurface layer. The presented antenna offers a significant potential for a number of platforms where the available space for antenna integration is limited, such as CubeSats, Smallsats, Rovers and Landers.

\section{REFERENCES}

[1] C. A Balanis, Antenna theory: analysis and design (John Wiley \& Sons, 2016).

[2] R. C Hansen, Phased array antennas, vol. 213 (John Wiley \& Sons, 2009).

[3] D. Schurig, et al, "Metamaterial electromagnetic cloak at microwave frequencies", Science, vol. 314, no. 5801, pp.977-980, 2006.

[4] D. R. Smith, D. Schurig, and J. B. Pendry, "Negative refraction of modulated electromagnetic waves," Applied Physics Letters, vol. 81, no. $15, \mathrm{pp} .2713-2715,2002$.

[5] J. B. Pendry, "Negative refraction makes a perfect lens," Physical review letters, vol. 85, no. 18, pp. 3966, 2000.

[6] D. R. Smith, J. B. Pendry, and M. C. Wiltshire, "Metamaterials and negative refractive index, Science, vol. 305, no. 5685, pp. 788-792, 2004.

[7] C. L. Holloway, E. F. Kuester, J. A. Gordon, J. O'Hara, J. Booth and D. R. Smith, "An Overview of the Theory and Applications of Metasurfaces: The Two-Dimensional Equivalents of Metamaterials," IEEE Antennas and Propagation Magazine, vol. 54, no. 2, pp. 10-35, April 2012.

[8] G. Minatti et al, "Modulated Metasurface Antennas for Space: Synthesis, Analysis and Realizations," IEEE Transactions on Antennas and Propagation, vol. 63, no. 4, pp. 1288-1300, April 2015.

[9] D. F. Sievenpiper, "Forward and backward leaky wave radiation with large effective aperture from an electronically tunable textured surface", IEEE Trans. Antennas Propag., vol. 53, no. 1, pp. 236-247, 2005.

[10] O. Yurduseven and D. R. Smith, "Dual-Polarization Printed Holographic Multibeam Metasurface Antenna," IEEE Antennas and Wireless Propagation Letters, vol. 16, pp. 2738-2741, 2017.

[11] M. C. Johnson, S. L. Brunton, N. B. Kundtz and J. N. Kutz, "Sidelobe canceling for reconfigurable holographic metamaterial antenna," IEEE Trans. Antennas Propag., vol. 63, no. 4, pp. 1881-1886, Apr. 2015.

[12] P. Sivaseetharaman, C. A. Balanis, and C. R. Birtcher, "Design of scalar impedance holographic metasurfaces for antenna beam formation with desired polarization", IEEE Trans. Antennas Propag. Vol. 63, 3016, 2015.

[13] S. Sun, Q. He, S. Xiao, Q. Xu, X. Li, and L. Zhou, "Gradient-index meta-surfaces as a bridge linking propagating waves and surface waves", Nat. Mater., vol. 11, pp. 426, 2012.

[14] A. M. Patel and A. Grbic, "A Printed Leaky-Wave Antenna Based on a Sinusoidally-Modulated Reactance Surface," IEEE Transactions on Antennas and Propagation, vol. 59, no. 6, pp. 2087-2096, 2011.
[15] S. Maci, G. Minatti, M. Casaletti, and M. Bosiljevac, "Metasurfing: Addressing waves on impenetrable metasurfaces," IEEE Antennas Wireless Propag. Lett., vol. 10, pp. 1499, 2011.

[16] M. Faenzi, et al, "Metasurface Antennas: New Models, Applications and Realizations," Scientific Reports, vol. 9, no. 1, pp.10178, 2019.

[17] O. Yurduseven, V. R. Gowda, J. N. Gollub and D. R. Smith, "Printed aperiodic cavity for computational and microwave imaging," IEEE Microw. Wireless Compon. Lett., vol. 26, no. 5, pp. 367-369, May 2016.

[18] O. Yurduseven, D. L. Marks, T. Fromenteze, and D. R. Smith, "Dynamically reconfigurable holographic metasurface aperture for a Mills-Cross monochromatic microwave camera," Optics Express, vol. 26, no. 5, pp.5281-5291, 2018.

[19] J. N. Gollub, et al, "Large metasurface aperture for millimeter wave computational imaging at the human-scale," Scientific Reports, vol. 7, pp. 42650, 2017.

[20] Y. Lee, S. J. Kim, H. Park, and B. Lee, "Metamaterials and Metasurfaces for Sensor Applications," Sensors, vol. 17, no. 8, pp. 1726, 2017.

[21] O. Yurduseven, T. Fromenteze, D. L. Marks, J. N. Gollub and D. R. Smith, "Frequency-Diverse Computational Microwave Phaseless Imaging," in IEEE Antennas and Wireless Propagation Letters, vol. 16, pp. 2808-2811, 2017.

[22] S. Mukherjee, Z. Su, L. Udpa, S. Udpa and A. Tamburrino, "Enhancement of Microwave Imaging Using a Metamaterial Lens," IEEE Sensors Journal, vol. 19, no. 13, pp. 4962-4971, 2019.

[23] D. L. Marks, O. Yurduseven, and D. R. Smith, "Cavity-backed metasurface antennas and their application to frequency diversity imaging," JOSA A, vol. 34, no. 4, pp.472-480, 2017.

[24] A. Darvazehban, S. Ahdi Rezaeieh, A. Zamani and A. M. Abbosh, "Pattern Reconfigurable Metasurface Antenna for Electromagnetic Torso Imaging," in IEEE Transactions on Antennas and Propagation, vol. 67, no. 8, pp. 5453-5462, Aug. 2019.

[25] O. Yurduseven et al., "Computational microwave imaging using 3D printed conductive polymer frequency-diverse metasurface antennas," in IET Microwaves, Antennas \& Propagation, vol. 11, no. 14, pp. 1962-1969, 19112017.

[26] A. Molaei, J. Heredia-Juesas, G. Ghazi, J. Vlahakis and J. A. Martinez-Lorenzo, "Digitized Metamaterial Absorber-Based Compressive Reflector Antenna for High Sensing Capacity Imaging," IEEE Access, vol. 7, pp. 1160-1173, 2019.

[27] D. R. Smith, et al, "An analysis of beamed wireless power transfer in the Fresnel zone using a dynamic, metasurface aperture," Journal of Applied Physics, vol. 121, no. 1, pp. 014901, 2017.

[28] A. K. Baghel, S. S. Kulkarni and S. K. Nayak, "Far-Field Wireless Power Transfer Using GRIN Lens Metamaterial at GHz Frequency," IEEE Microwave and Wireless Components Letters, vol. 29, no. 6, pp. 424-426, 2019.

[29] V. R. Gowda, M. F. Imani, T. Sleasman, O. Yurduseven and D. R. Smith, "Focusing Microwaves in the Fresnel Zone With a CavityBacked Holographic Metasurface," IEEE Access, vol. 6, pp. 12815$12824,2018$.

[30] M. Ettorre, R. Sauleau and L. Le Coq, "Multi-beam multi-layer leakywave SIW pillbox antenna for millimeter-wave applications," IEEE Trans. Antennas Propag., vol. 59, no. 4, pp. 1093-1100, Apr. 2011.

[31] D. R. Smith, O. Yurduseven, L. P. Mancera, P. Bowen, and N. B. Kundtz, "Analysis of a waveguide-fed metasurface antenna," Physical Review Applied, vol. 8, no. 5, pp. 054048, 2017.

[32] O. Yurduseven, D. L. Marks, J. N. Gollub, and D. R. Smith, "Design and analysis of a reconfigurable holographic metasurface aperture for dynamic focusing in the fresnel zone," IEEE Access, vol. 5, pp. 15055-15065, 2017.

[33] L. Di Palma, et al, "Circularly-Polarized Reconfigurable Transmitarray in Ka-Band With Beam Scanning and Polarization Switching Capabilities," IEEE Transactions on Antennas and Propagation, vol. 65, no. 2, pp. 529-540, 2017. 